\title{
Detection of Ridge Discontinuities in Fingerprint Recognition Influenced by Skin Diseases
}

\author{
Mona Heidari and Martin Drahansky \\ Brno University of Technology, Faculty of Information Technology, Centre of \\ Excellence IT4Innovations, Bozetechova 2, 612 66, Brno, Czech Republic \\ iheidari@fit.vutbr.cz,drahan@fit.vutbr.cz
}

\begin{abstract}
This paper presents a new approach to detection and classification of skin diseases in fingerprints using these methods: Thinning Algorithm, Thresholding, Block Orientation Field. The experimental results show the method is obtained from combination of the mentioned algorithms and is able show the damaged areas in fingerprints.
\end{abstract}

Keywords: Fingerprint recognition, skin diseases, image processing, image segmentation, computer vision

\section{Introduction}

Fingerprint-based systems are the most widely used biometric technology. Although the individuality of fingerprints was well known already in the ancient times, it was not until 1880 when Henry Faulds published a work that introduced the possibility of using fingerprints for the purpose of human identification [1]. Since that time, fingerprint technology has been evolving and nowadays fingerprint recognition systems have been applied in a variety of areas [2]. They are used not only in forensics for crime purposes but also as an access method to facilities, computers, mobile phones or electronic banking; as a data protection method and for civil identification (passports, driver licenses, national IDs), not to mention applications in government, commercial financial sector, education or health care [2]. This technology has been well accepted by people and we use it on a daily basis.

However, there is a significant number of people who cannot use fingerprint systems as easily because their fingertip skin is affected by some kind of skin disease. As these systems count heavily on the structure of an individual's fingertip papillary line pattern that positively determines their identity, people suffering from skin diseases might be discriminated against as their papillary patterns may be impaired. It is very likely that fingerprint devices have not been designed to deal with damaged fingerprints, and therefore after scanning the fingerprint, they usually reject it.

However, nowadays skin diseases which influence fingerprints are becoming increasingly notable. There is a large number of skin diseases that affect the skin in some way. Generally speaking, these diseases can be classified into three groups: diseases causing histopathologic changes of the epidermis and dermis, diseases causing skin discoloration and diseases causing histopathologic changes in of the epidermis and dermis and skin discoloration. Diseases from the first group cause problems to all types of fingerprint scanners because they affect both the color and the internal structure of the epidermis and dermis [3].

Once the papillary line structure has changed, it is difficult to recognize the original pattern and therefore often impossible to determine a person's identity. Among diseases in this group are for instance hand eczema, dyshidrosis, systemic sclerosis or Raynaud's

Received (January 16, 2018), Review Result (April 3, 2018), Accepted (April 8, 2018) 
phenomenon [3]. Diseases causing histopathological changes in the junction between epidermis and dermis also belong to the first group.

Fingerprint recognition is not as simple process as it might look. Starting from the acquisition of a fingerprint image, called a sample, there are a number of challenges along the way. Fingerprint recognition systems are never $100 \%$ precise and the quality of their results sometimes cannot be compared to the work of a forensic expert. However, they bring many advantages, such as invaluable speed of processing and ease of storage.

There are two types of biometric systems: a verification system or an identification system [2]. The purpose of the former is to authenticate a person's identity by comparing their sample to the one that was captured previously. The latter recognizes a person by going through the whole database to find a match. Regardless of whether we want to verify or identify a person, or even capture and store a sample and data of a completely new person's identity, in all cases the samples taken are involved in a similar fingerprint recognition process. The main steps of the process are as follows:

1. Fingerprint acquisition. For capturing the digital image of a person's papillary lines structure, there is a wide range of fingerprint sensors to choose from, plus a traditional off-line ink or clean fingerprinting method [2]. As the quality of the sample is very important for the fingerprint recognition, it is necessary to choose a high quality sensor [4].

2. Fingerprint enhancement. After a sample is acquired, pre-processing image enhancing steps improve the papillary lines structure for the following image processing, classification and matching. However, the enhancement steps differ for every fingerprint sensor and the results depend both on the environment conditions in which the sample was captured and on possible skin damage, skin humidity or dryness, or even dirt present on the finger.

3. Fingerprint classification. Every fingerprint is assigned to one of fingerprint classes according to its external shape. For this purpose, so called singular points, loop and delta, are useful [2]. This step speeds up the identification process because after determining the class, the fingerprint does not have to be compared with an entire database of fingerprints but only with fingerprints in that particular class. However, it is a demanding process, because due to the variability of the fingerprint patterns, it is often difficult to determine which class the fingerprint belongs to [2] [4].

4. Minutiae extraction. Minutiae are significant points in the papillary line structure. The combination of their positions and types is unique for each person and therefore is used for representing their identity. There are over 150 types of minutiae [2] but all of them consist of two basic ones: the ridge ending and ridge bifurcation. This step can be problematic in low-quality fingerprints, for example those affected by some kind of a skin disease. The whole process of minutiae extraction is displayed in Figure 1 and 2.

5. Fingerprint matching. In this step, sets of minutiae from two fingerprint images, the template and the input, are compared. The result is a number of corresponding pairs of minutiae. After the comparison it can be stated whether or not the two fingerprints belong to the same person [2]. 


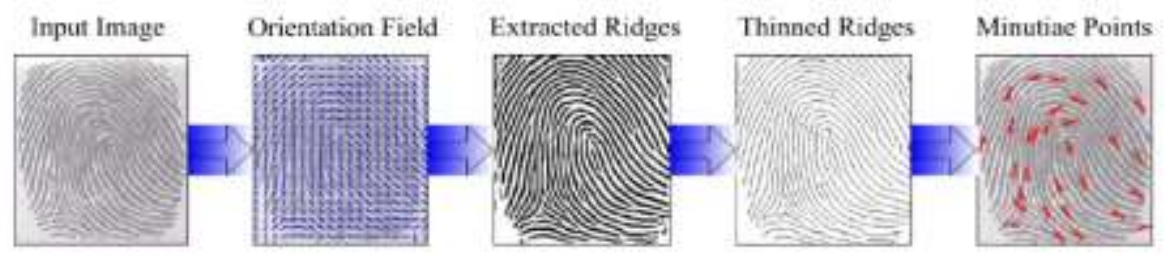

Figure 1. The Minutiae Extraction Algorithm [4]

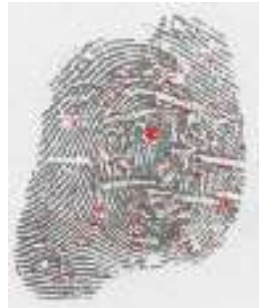

Figure 2. Minutiae Extraction of a Fingerprint Affected by Eczema

In order to obtain the structure of a person's papillary lines in the form of a digital image, various sensing mechanisms can be used. There are two primary methods of a capturing a fingerprint image: Inked (off-line) and Live scan. Fingerprint scanners can be either singlefinger or multi-finger. As the name suggests, only one finger can be scanned at a time using a single-finger scanner, whereas multi-finger scanners usually allow us to scan four fingers at once. The general structure of a fingerprint scanner consists of a sensor that reads the ridge pattern of the finger and an $\mathrm{A} / \mathrm{D}$ converter that converts the analog signal to the digital form. An interface module then communicates with external devices, such as a computer [2].

In this work we used a database contains large volume of diseased fingerprint images from patients suffering from various kinds of skin diseases which is acquired in cooperation with medical experts, which are collected and stored to be used in our laboratory.

The work introduced in this paper combines the different algorithms for localizing the damage and show the extent of damage.

\section{Fingerprint Image Enhancement Methods}

Fingerprint Identification is one of the most well-known biometric technologies, because of their uniqueness and consistency over time, fingerprints have been used for identification over a century more recently becoming automated due to advancements in computing capabilities. Fingerprint images are acquired and stored into the database in the image acquisition stage. These images are then enhanced in the image processing stage by performing gray level enhancement, spatial filtering, image sharpening, edge detection, segmentation and thinning processes.

Some Enhancement technique is needed and essential in order to prepare the images for further measurement. The quality of fingerprint images should be reduced due to noise, skin conditions, etc. Generally, for a given digital fingerprint image, the region of interest can be divided into the following three groups [5]:

- Well-defined region, where ridges and valleys are clearly differentiated from one another such that a minutiae Extraction algorithm is able to operate reasonably.

- Recoverable corrupted region, where ridges and valleys are corrupted by a small amount of creases, smudges, etc. But, they are still visible and the neighboring regions provide sufficient information about the true ridge and Valley structures. 
- Unrecoverable corrupted region, where ridges and valleys are corrupted by such a severe amount of noise and distortion that no ridges and valleys are visible and the neighboring regions do not provide sufficient information about the true ridge and valley structures either.

The goal of an enhancement algorithm is to improve the clarity of papillary line structures of fingerprint images in recoverable regions and to remove the unrecoverable regions. In low quality fingerprint images, papillary lines structures, are not always welldefined therefor they cannot be correctly detected. This leads to following problem:

1. A notable number of false minutiae may be created.

2. A large percent of real minutiae may be ignored.

3. Large errors in their position and orientation, may be introduced.

Fingerprint enhancement can be conducted on either binary images or gray level images. A binary ridge image is an image where all the ridge pixels are allocated a value one and valley pixels are allocated a value zero. However, after applying a ridge extraction algorithm on the original gray-level images, information about the true ridge structures is often lost depending on the performance of the ridge extraction algorithm. Therefore, enhancement of binary ridge images has its inherent limitations. In a gray-level fingerprint image, ridges and valleys in a local neighborhood form a sinusoidal-shaped plane wave which has a well-defined frequency and orientation. The principle objective of gray level enhancement is to improve the contrast of the fingerprint image because the acquired fingerprint image in the earlier session might be too bright or too dark.

Fingerprint enhancement algorithm includes the following:

- Normalization

- Segmentation

- Orientation Estimation

- Ridge Frequency Estimation

- Gabor Filtering

- Binarization and Thinning

In this work we used some enhancement techniques in order to separate images into background and foreground regions therefore we obtained clear fingerprint area containing the ridges and valleys. Segmentation is one of the steps through this goal.

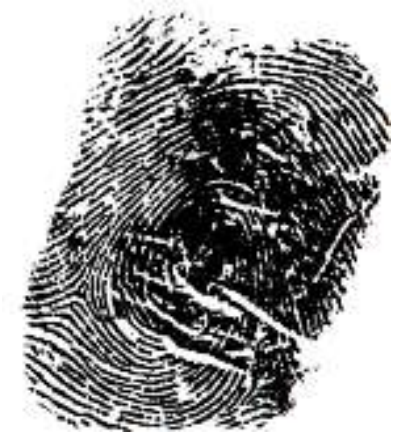

Figure 3. Segmentation Result of Fingerprint from Database

The main objective of segmenting a fingerprint image is to separate the clear fingerprint area from the non-fingerprint area. In this stage, the edge detected gray level fingerprint 
image is threshold to a binary image by using adaptive thresholding methods. In this case we applied k-means algorithm. The background corresponds to the regions outside the borders of the fingerprint area, which do not contain any valid information. When minutiae extraction algorithms are applied to the background regions of an image, it results in the extraction of noisy and false minutiae. In a fingerprint image, the background regions generally exhibit a very low grey-scale variance value, whereas the foreground regions have a very high variance. Hence, a method based on variance thresholding can be used to perform the segmentation.

The main idea in threshold methods is to select a threshold $\mathrm{T}$ that can separate objects from the background. This threshold can be specified according to the intensity histogram. Histogram of an image displays the gray-level values versus the number of pixels at that value. For fingerprint images, the histogram shows the contrast of the image and the distribution of the gray level as shown below in Figure 4.

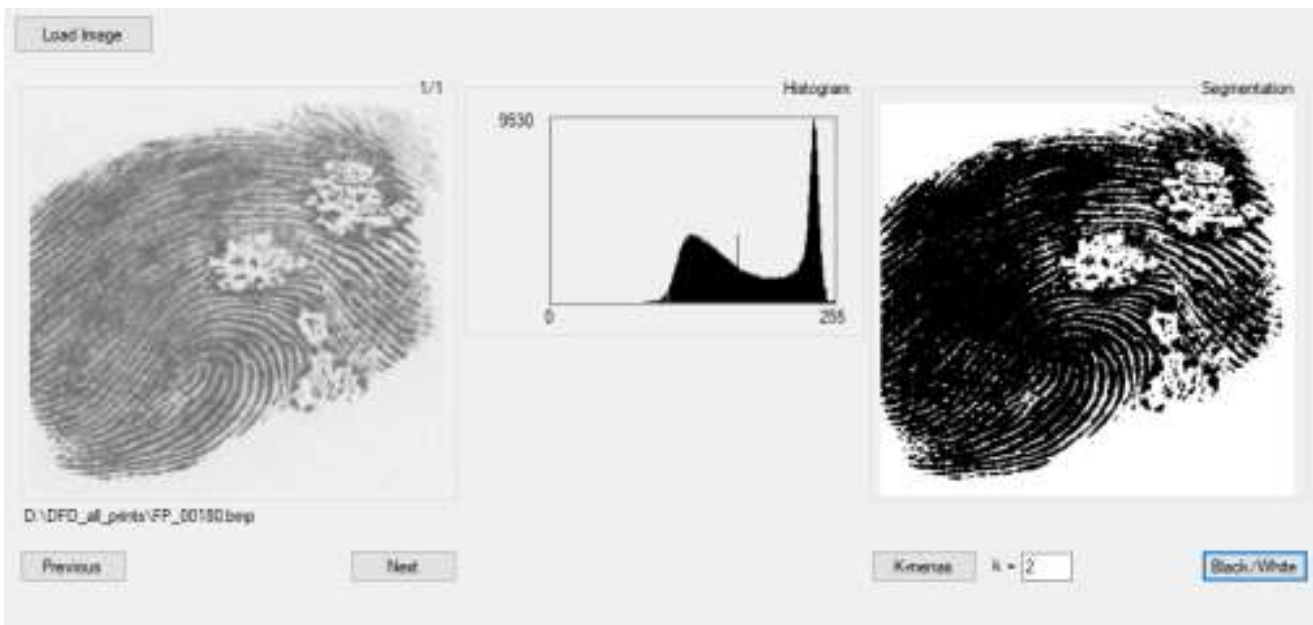

\section{Figure 4. GUI of The Application Which Shows Histogram Plot and Result of Fingerprint Segmentation}

In our application we used image enhancement technique to improve the image quality and to make it clearer for further operations. Often fingerprint images from various sources lack sufficient contrast and clarity. It increases the contrast between ridges and furrows and connects the some of the false broken points of ridges due to insufficient amount of ink or poor quality of sensor input, as it is shown in right side of the image in Figure 4.

We have implemented histogram analysis. A histogram computed for any fingerprint image is similar; it contains a high number of light pixels and a high number of dark pixels, greyscale values are present less frequently. The histogram image has therefore a bi

modal shape: two similarly high peaks and one valley in between them. Both from the characteristics of a fingerprint image and by practical experimenting we observed that a histogram computed for any region of interest from the fingerprint area (the region must not be partially in the fingerprint area and partially in the background) has to have similar characteristics, provided that it belongs to a healthy fingerprint. The reason for it is that an ideally healthy fingerprint is characterized by smooth dark ridges and clear light valleys, both having a similar width. They represent the two high histogram values and the border, as a gradual transition between these colors, represents the greyscale values between the two peaks in the histogram.

However, the lower the quality, the less the histogram resembles the ideal bimodal histogram. Although a damaged ROI (Region of Interest) does not necessarily imply a nonbimodal histogram, as a histogram generally only provides an information about intensity 
distribution of an image, a non-bimodal histogram always implies a damaged or a lowquality area.

Along with histogram analysis we have histogram equalization which is a technique of improving the global contrast of an image by adjusting the intensity distribution on a histogram. This allows areas of lower local contrast to gain a higher contrast without affecting the global contrast. Histogram equalization accomplishes this by effectively spreading out the most frequent intensity values.

\section{Influence of Skin Diseases}

Skin diseases represent very important, but often neglected factor of the fingerprint acquirement. It is impossible to say in general how many people suffer from skin diseases, because there are so many various skin diseases [6].

There are a lot of skin diseases, which can affect palms and fingers. These diseases can be divided into two groups. In the first group, palms and fingers are mainly affected. In the second group, skin lesions can be diffuse, involving wide areas. We can also differentiate skin diseases [7] [8], by type or localization of histopathological changes in epidermis and dermis.

If the diseases has attacked and destroyed the structure of papillary lines in the epidermis and underlying dermis (top two layers of the skin), the papillary lines will not grow in the same form as before (if at all) and therefore such user could be restricted in their future life by being excluded from the use of fingerprint recognition systems, though their fingers do not have any symptoms of the skin disease anymore [3].

Some of these diseases are only temporary; that is, after the healing of such disease, the papillary line structure or color is restored and the user is again able to use their fingers for the fingerprint recognition for the authorization tasks in security systems. However, some diseases leave irrecoverable finger damage restraining new growth of papillary lines, and respective user is then unable to use their fingerprints for appropriate recognition tasks in automated fingerprint security systems [7] [8]. In some cases the condition of the image obtained from the damaged fingerprint is not even good enough for further processing, but in others the damage is minor and the condition of the fingerprint image should not be an obstacle for papillary lines and minutiae extraction, and further matching.

\section{Proposed Method for Localizing Damaged Area}

The raw diseased fingerprint database was first analyzed in order to provide a solid foundation for future research. For every particular disease, common signs among all fingerprint images affected by this disease were found and a general description of each disease and its influences was defined.

Based on these descriptions and sets of common signs and their frequencies, the diseased fingerprint images were classified into 5 categories. These categories are later used in the actual detection of the damaged areas in a fingerprint image and they help to divide the large detection task into smaller bearable parts.

Most of the fingerprint images come from a dactyloscopic card. By observing and comparing the fingerprint images, 12 common features were defined.

7 of them are local features:

- straight lines (SL)

- a grid (G)

- small papillary lines disruptions (PLD),

- small "cheetah" spots (CS),

- larger round/oblong spots (ROS), 
- large irregular spots (IS) and

- dark places (DP).

The other 5 were global image patterns:

- blurriness of (parts of) the image (B),

- a significantly high contrast of the image (HC),

- the entire fingerprint area affected (EA),

- total deformation of the fingerprint image (TD) and

- a significantly high quality and healthy fingerprint (HQ).

The proposed damage localization method consists of the following major steps: Thinning, Thresholding and Orientation Field. The combination of these methods bring the useful information about the damaged area on fingerprints. Based on the database analysis, an application capable of detecting damage area and determine the quality of the fingerprint image for further processing.

\subsection{Results of the Thinning Algorithm}

In this stage we need to partition the image into a foreground and background which isolates objects by converting grayscale images into binary images. Black pixels correspond to background and white pixels to foreground. Indeed, the segmentation is determined by a single parameter known as the intensity threshold that means every pixel in the image is being compared with this threshold. If the pixel's intensity is higher than the threshold, the pixel is set to, for example, white in the output, otherwise if it is less than threshold, it is set to black. After segmentation, we use thinning algorithm to lines of a single pixel thickness and reduce the thresholded output of an edge detector, "the Sobel operator" which we are used it in the next step.

There in Figure 5 (a) result of thinning on a healthy fingerprint and (b) fingerprint affected by a Hyperkeratotic eczema.

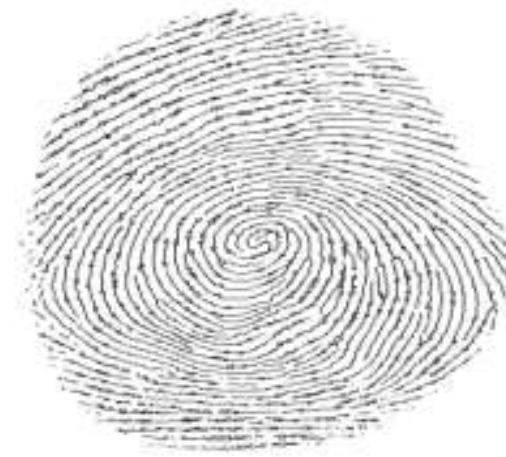

(a) Healthy Fingerprint

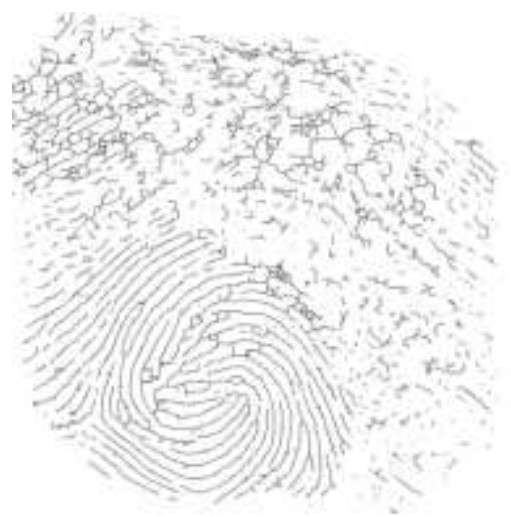

(b) Hyperkeratotic Eczema

Figure 5. Result of the Thinning Algorithm on the Healthy and Fingerprint Affected by a Skin Disease

\subsection{Ridge Discontinuity Detection based on Orientation Estimation}

For the purpose of indicating the direction of ridges and classifying the fingerprint image into one of the several fingerprint classes the computation of block orientation field is commonly used. 
For extracting local ridge orientation the most natural approach is based on computation of gradients in the fingerprint image [2]. The information of fingerprint which is consists of dark and white lines can be easily processed by a gradient operator that estimates the image gradient for each pixel. This low-level information is gathered and averaged for each $\omega \times \omega$ block in the image [9]. The result for a healthy fingerprint shows a smooth and continual image of the ridges direction estimates - see the Figure below. According to this and the following information, not only discontinuities in the block orientation field but even universal abnormalities can be found via this method.
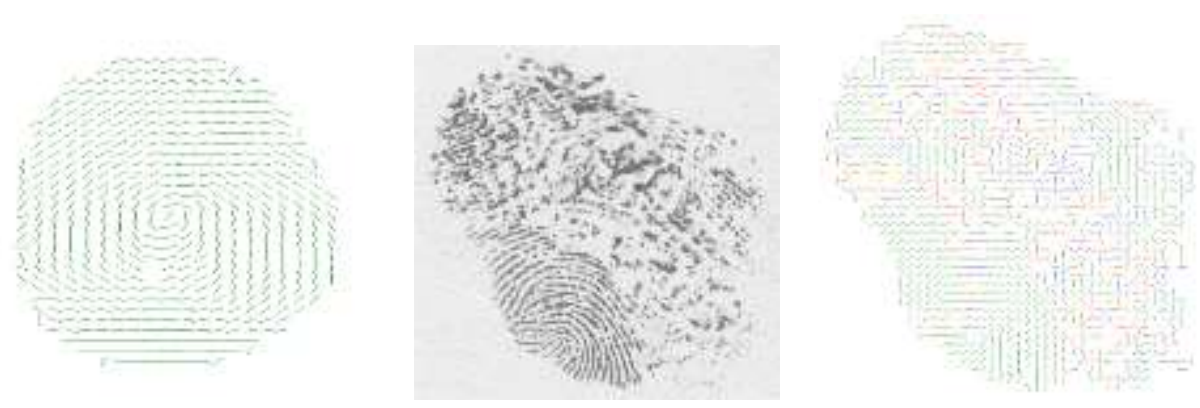

\section{Figure 6. Examples of Orientation Estimation Images (Healthy Fingerprint, Fingerprint Affected by a Skin Disease, Detected Damaged Areas)}

As we see in Figure 6 the result of the block orientation field for a damaged fingerprint will be discontinuous in devastated areas. The main steps of the gradient-based method of block orientation field are as follows [2]:

1. Compute the gradients $\partial_{x}(i, j)$ and $\partial_{y}(i, j)$ at each pixel of the fingerprint image $(i, j)$.

2. 2. Divide the original fingerprint image into blocks of size $\omega \times \omega$.

3. Compute the estimation $\theta(i, j)$ of the ridge orientation of each block centered at $(i, j)$ using the following equations:

$$
\begin{aligned}
& v_{x}(i, j)=\sum_{u=i-\frac{w}{2}} \sum_{v=j-\frac{w}{2}}^{u=i+\frac{w}{2} v=j+\frac{w}{2}} 2 \partial_{x}(u, v) \partial_{y}(u, v) \\
& v_{y}(i, j)=\sum_{u=i-\frac{w}{2}}^{u=i+\frac{w}{2} v=j+\frac{w}{2}} \sum_{v=\frac{w}{2}} \partial_{x}^{2}(u, v) \partial_{y}^{2}(u, v) \\
& \theta(i, j)=\frac{1}{2} \tan ^{-1}\left(\frac{v_{y}(i, j)}{v_{x}(i, j)}\right)
\end{aligned}
$$

Where $\theta(i, j)$ is the least square estimate of the local ridge orientation at the block centered at pixel $(i, j)$.

The least mean square estimation method is used to compute the orientation image. The resulting block orientation field is analyzed for any discontinuities that may occur. In this case the analysis of estimating the orientation block-wise is done based on using a pixelwise scheme (a row-wise and column-wise scanning) which produces a finer and more accurate estimation of the orientation field and reveals areas of possible damage in the 
fingerprint. Adjacent blocks' directions are compared and if $\mid \theta(i, j)-\theta\left(i, j+1 \mid>45^{\circ}\right.$ then a block is marked as a discontinuity, where estimating of $\theta(i, j)$ and $\theta(i, j+1)$ have a value between $0^{\circ}$ and $180^{\circ}$.

This algorithm provides, justly precise estimate of the fingerprint image. The main advantage of this algorithm is that it is a part of standard fingerprint recognition pipeline then it can be easily implemented into existing method.

\section{Fingerprint Image Quality Estimation}

The purpose of combination of these methods, is to identify the range of damage and grade bad blocks, and that make it possible to decide whether any subsequent analysis of the fingerprint is feasible.

The result of each method, first is called and saved individually into an $n \times n$ array of integers this array is exactly as the same size as the input image. The next step is combining these three arrays due to the labeling the pixels of image which " 0 " shows background pixels so background array contains this pixel and obviously it is considered a background, "1" means pixels are fine and the array clearly includes pixels belong to the healthy area of an image and if a pixel labeled "2", in each of two first arrays it is counted damaged.

\section{Experimental Results}

The proposed methodology is tested on our database of diseased fingerprint consists of approximately 900 samples of dactyloscopic cards. These images had already been classified into diseases classes by dermatologists (medical specialists).

\section{Conclusion}

Results show that the proposed method detects almost all anomalies in fingerprint images and the method detects of areas influenced by skin diseases in the fingerprint. Elimination of noise in the images should be completely removed so that the system can reliably detect the damaged area.

Future work of this project would include the creating of the algorithm to classify fingerprint diseases that would be a combination of the results of this work with the new algorithms. Methods are ready to be enhanced and further developed. There are opportunities for the results of the future work to be used in medical applications or program for police department and security purposes.
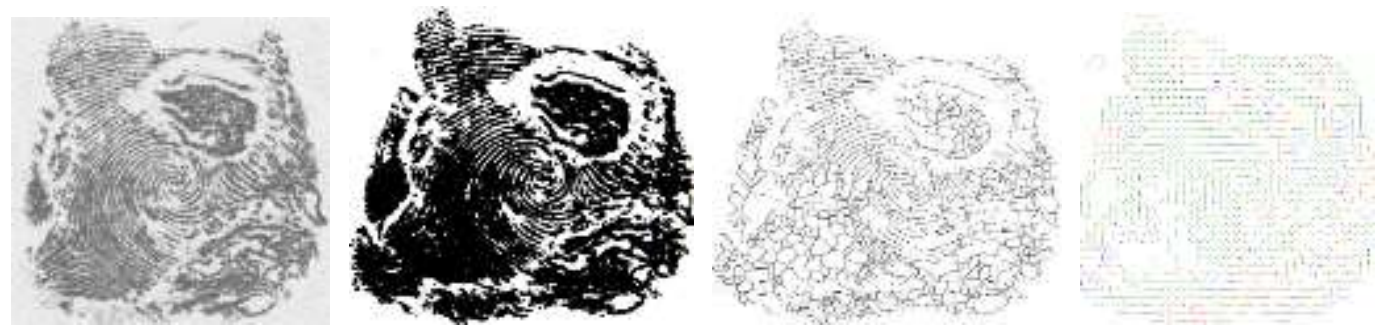

Figure 7. Examples of Processed Image Affected by Psoriasis Vulgaris (Original image, Thresholding, Thinning, Orientations)

\section{Acknowledgements}

This work was supported by The Ministry of Education, Youth and Sports of the Czech Republic from the National Programme of Sustainability (NPU II); project IT4Innovations excellence in science - LQ1602 and the BUT project Secure and Reliable Computer Systems FIT-S-17-4014. 


\section{References}

[1] D. M. O. F. Drahansky M., "Biometrie”, Springer, pp. Dermatologic diseases and fingerprint recognition, In Y. Zhang, A. Cuzzocrea, J. Ma, K.-i. Chung, T. Arslpages, (2011), pp. 251-257.

[2] M. D. A. K. P. S. Maltoni Davide, "Handbook of Fingerprint Recognition", Springer Publishing Company, Incorporated, (2009).

[3] D. M. U. J. B. E. T.-h. Dolezel Michal, "Influence of Skin Diseases on Fingerprint Quality and Recognition", InTech, (2012).

[4] M. Drahansk, "Fingerprint Recognition Technology - Related Topics", saarbrucken: Lambert Academic Publishing, (2011).

[5] N. a. B. R. Ratha, "Automatic Fingerprint Recognition Systems”, Springer New York, (2003).

[6] F. P. R. A. A. Jain Anil K., "Handbook of Biometrics", Springer-Verlag New York, Inc., (2007).

[7] M. a. H. D. a. B. E. Drahansky, "Fingerprint Recognition Influenced by Skin Diseases", International Journal of Bio-Science and Bio-Technology, (2010).

[8] B. E. D. M. O. Filip., "Dermatologic Diseases and Fingerprint Recognition”, Springer Berlin Heidelberg, pp. 251-257.

[9] L. H. a. Y. W. a. A. Jain, "Fingerprint image enhancement: algorithm and performance evaluation", IEEE Transactions on Pattern Analysis and Machine Intelligence, (1998).

\section{Authors}

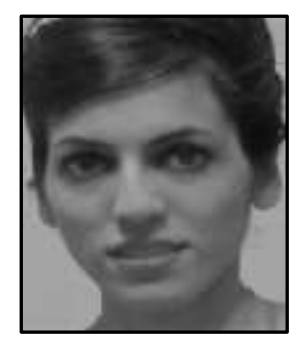

Mona Heidari, works as Ph.D. student at the Brno University of Technology, Faculty of Information Technology, Department of Intelligent Systems. Her research topics include biometrics, Computer vision and Deep learning.

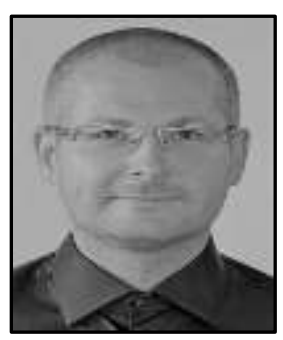

Martin Drahanský, graduated in 2001 at the Brno University of Technology, Faculty of Electrotechnics and Computer Science in the Czech Republic and simultaneously at the FernUniversität in Hagen, Faculty of Electrotechnics, Germany. He achieved his Ph.D. grade in 2005 at the Brno University of Technology, Faculty of Information Technology in the Czech Republic. Now he works as associate professor at the BUT FIT, DITS. His research topics include biometrics, security and cryptography, artificial intelligence and sensoric systems. For more information - see please http://www.fit.vutbr.cz/ drahan/. 